\title{
Survey on Mobile Edge Computing for Internet of Things System
}

\author{
Keerthivasan $\mathrm{K}^{\mathrm{a}, 1}$, and Shibu $\mathrm{S}^{\mathrm{b}}$ \\ ${ }^{a} P G$ Scholar, Dept of ECE, Panimalar Engineering College, Chennai \\ ${ }^{b}$ Associate professor, Dept of ECE, Panimalar Engineering College, Chennai
}

\begin{abstract}
IOT is the latest advanced technology. To help the customer value, it is the backbone of both service sector and product area businesses. In $5 \mathrm{G}$ technology, internet of things is a main role to play in future. It is used to support the intelligent, big data analytics and lots of application such as health care, smart cities and industrial. Advancement of 5G technology will recognize MECfor future of cloud computing. MEC is used to provide multiple application such as service providers, mobile subscribers, enterprises and other vertical segments. MEC technology provides low latency and quality of services [QOS].It distributes the network uniformly with strong mobility supports. In this paper a detailed review of MEC technology for IoT application is discussed.
\end{abstract}

Keywords. Mobile Edge Computing (MEC), Internet Of Things (IOT), virtualization, mobility management, security.

\section{Introduction}

\subsection{IoT-Overview}

For the past four years, the Internet of Things is rising and leading in different areas of our day today life [e.g. healthcare, transportation, vehicle etc].IoT exposed as a vast prototype combining adaptable and epic group of smart things starting from the internet. IoT systems are needed computing resources, sufficient data and network structures to accommodate more interconnected devices. The existing cellular networks need to be modified to meet these enormous demands.For $5 \mathrm{G}$, we need adequate mobile network with more bandwidth $[10 \mathrm{gbps}]$, low latency $[1 \mathrm{~ms}]$ and very low cost. The formation of Heterogeneous Network (HetNet) into the cellular system improves the overall spectral efficiency and network capacity of the system[1].This enables more quality of service [QOS] and quality of experience [QOE] for IOT services. More smart devices such as phones, personal computers and vehicles connected under a Internet. It improves the machine to human and human to machine communication. Focusing on high opportunity, accuracy and security[2].

${ }^{1}$ Keerthivasan $\mathrm{K}^{1}$,PG scholar ,dept of ECE , Panimalar Engineering College, Chennai, India Email:keerthivasan.k21@gmail.com 


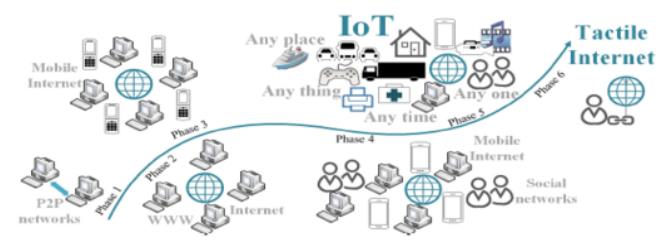

\subsection{MEC:}

Figure 1. Evolution Of The Internet ${ }^{[1]}$.

The Mobile Edge Computing [MEC] is conditionally delivering the computing and the storage capacity from remote input centers to that mobile edge in procedure to trim the network prevent between end equipment and then computing resources. Permit IoT with Mobile Edge Computing can permanently develop the quality of services for IoT operations. Mostly IoT applications that try to access the corresponding inputs from various types of IoT objects and develop high-level ability by considering the acquired input based on the data systematic models[3]. The MEC performing cloud computing functionalities at the edges of the cellular networks. Hence, the congestion in the network reduced and improvise the resource optimization, user viewing experience and the entire network performance. MEC will reduce the latency and spectrum utilization of the wireless network which helps to service providers and application developers. Normally, cloud computing initiates the outsourcing of processing functionalities and storage of IoT data to a external source for to minimize the complexity in the self-management functions and data security. However, the conventional cloud servers are centralized in nature, which faces multiple issues such as the failure in the nodes, minimal local awareness, availability, and latencies in the Wide Area Networks(WANs). Although, several IoT applications required decentralized systems which required location awareness, minimal latency ,scalability, geo-distribution and mobility management. Edge computing proposed to minimize the distance between the IoT devices and centralized cloud[4].

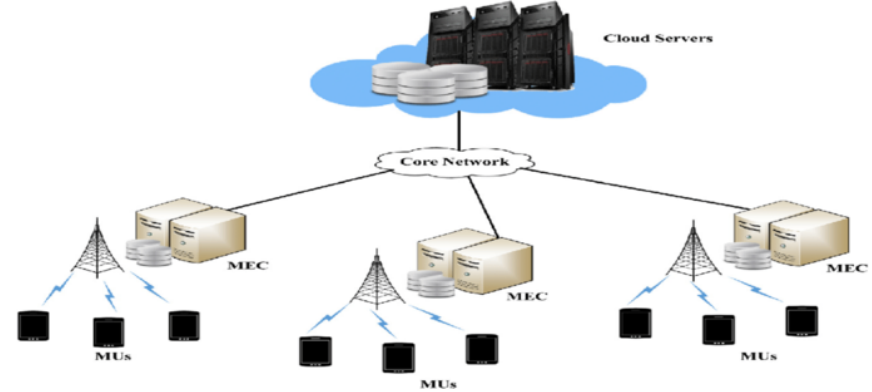

Figure 2. Mobile Edge Computing Architecture.

The important analysis of mobile edge computing is to deal with the objections of greet from MCC scheme. Mobile Edge Computing permit Mobile Country Code by increasing cloud resources

\section{IOT and MEC Application Scenarios}

\subsection{Smart home and Smart city}

Home applications that are based on IoTidea that are now accessible in most shopper markets. As an answer, MEC use particular and solid nearby administrations for preparing and capacity abilities for the huge IoT traffic made inside a structure. The 
customary doors which permit IoT applications to run on the brought together cloud can be enabled with MEC-server functionalities [5]. IoT innovation has progress from home to network.We see various future guarantees for open wellbeing, human services, utility, the travel industry, and the vehicle divisions.low latency and location awareness.

\subsection{Healthcare}

The important cases of $5 \mathrm{G}$ isrecognized as mobile health and telemedicine.Theelderly person sitting next to humanoid robots need some tactile feedback for $1 \mathrm{~ms}$ latency for their taking care service. In e-Healthcare applications,edge computing performance in big data is important. Although the IoT it widely used in health sector \& without edge computing it is difficult to achieve its performance and goals like MEC[6][17].

\subsection{Vehicle to Everything communication with IOT}

Vehicle to everything communication is done in many concepts mainly in vehicle to vehicle communication, home, grid and infrastructure. It provides low latency, good throughput and reliability [7].It improves the performance by RAN technology in IoT application. The MEC place a vital role to improves the vehicle to vehicle communication with IoT.

\subsection{Wearable in IOT (WIOT)}

Wearable IoT technology is used for the applications such as step trackers, smart watches, glasses. Low power technology is uses the technique such as Bluetooth low energy [BLE]. The main advantage is the throughput is obtained in gigabits. It is used in smart cities to increase the network efficiency and network traffic to provide good service quality. Virtual reality and Augmented reality works in only gigabits which is found in this technique[8].[16]

\subsection{Gaming for $A R$ and $V R$}

It is used for data aggregation IoT devices. It is used in many ways like tourism, telepresence, robotic assisted surgeries and smart transportation network. The interaction time between human and machines requirement should be less than $1 \mathrm{~ms}$. But the latency should be more than $1 \mathrm{~ms}$. It improves the efficiency in the network and overall performance is improved. In the virtual world data is aggregated to the IoT devices [9].

\section{Technical Aspects of MEC Enabled IOT}

\subsection{Scalability}

The scalability is the important factor in the actual utilization of MEC for IoT services. The suitability of MEC servers to various network conditions is the key factor for its adoption in next generation networks. The IoT system consist of sensors, Radio-Frequency Identification tags, actuators, vehicles, embedded systems and software all connected in a single network. These all devices are jointly works and provide reliable services for various technologies such as smart homes, smart grids, virtual power plants, smart cities and, intelligent transportation.More the number of devices connected in an IoT environment the efficiency is improved in the network space. The Radio-Frequency Identification-tags are found in each object to sense theefficiency. This application is usedinspite of changing the data size and user volume data, the information is unchanged and meets the user need[10]. 


\subsection{Mobility Management}

In MEC, the QoS is degraded, if mobile user elements(UEs) are moving away from the computing node. Hence, mobility management is a crucial factor in the MEC network.The UEs are disconnected from MEC network due to severe QoS degradation. The major goal of the MEC network is to provide more reliable mobility management scheme for IoT services.IP networks are security control for applications on the move. LTE is used for roaming, moving network and vertical transfers for more efficiency. The goal of energy aware Mobility management (EMM) is to optimize the downloaddelay resulting from both radio access and computation, under the user's con straint on long-term energy consumption. The moving nodes are adjusted based on the coverage area to the transmitted power[11].

\subsection{Security}

In IOT systems, the major security issues found on mobile networks, sensor networks and the entire Internet [12]. The security threats majorly targeting the MEC nodes, i.e., the MEC server and connected IoT nodes. Mobile Edge Computing based on IoT provides greater security, quality and development facility. The overall performance is improved by overcoming the threats like Denial of service, Man in the middle and heterogeneous network attack. Wireless local area network applications used for IoT security.

\subsection{Transfer of Resource intensive computational task}

It is the most popular topic discussed in IoT devices it has expanded the computing capability [13]. The nodes are very hard to reach the application so more regular offloading data is found. The low power wireless system is used in IoT network has Zigbee and Wi-Fi. The IoT devices are accessed by IoT gateway to reach the mobile edge computing server[17].

\subsection{Privacy}

The modern IoT systems are larger network spanning across countries and continents and adapted to various regulations. Similarly, privacy is key requirement in the healthcare applications. In an IoT network the data are shared and processed in the cloud, that could increase the privacy concerns. The effective usage of data in the unpredicted applications may sacrifice the privacy [14].

\subsection{Trust management}

Trust management is related to the total security of the cellular network or the working platform. It is very critical in the $5 \mathrm{G}$ applications like autonomous vehicles, remote surgeries, drones and factory automation. In these conditions, reliability and latency are very important parameters[15]. Normally, crucial applications are offloaded to the edge of the cloud which is not controlled by the client. Hence effective Trust management scheme required for IoT systems to achieve the desired results.

\section{Conclusion}

The advances in MEC and IoT technology would make an enormous contribution to achieving the future generations. MEC is the recent technology is used with IoT to 
bridge between low latency and huge IoT network. MEC uses cloud computing devices based on IoT to improve network heterogeneity, scalability, usability and protection against present challenges. MEC and IoT are the two technology which has the ability to accelerate to performance of the $5 \mathrm{G}$ network.

\section{References}

[1] S.Shibu and V.Saminadan, Enhanced interference cancellation techniques fordownlink of LTE-A heterogeneous networks .Int. J. Wireless and Mobile Computing, Vol. 17, No. 2, 2019.

[2] Survey on Multi-Access Edge Computing for Internet of Things Realization IEEECommunicationsSurveys\&Tutorials ( Volume: 20, Issue: 4, Fourthquarter 2018 ) 21 June 2018 PawaniPorambage ; Jude Okwuibe_; MadhusankaLiyanage; Mika Ylianttila_; TarikTaleb_

[3] A. M. Rahmani, T. N. Gia, B. Negash, A. Anzanpour, I. Azimi, M. Jiang, and P. Liljeberg, "Exploiting Smart e-Health Gateways at the Edge of Healthcare Internet of Things: A Fog Computing Approach," Future Generation Computer Systems, vol. 78, pp. 641-658, 2018.

[4] "ETSI executive briefing - mobile edge computing (mec) initiative," https://portal.etsi.org/portals/0/tbpages/mec/docs/mec\%20executive\% 20brief\%20v1\%2028-09-14.pdf, accessed on 01.02.2018.

[5] V. Frascolla, F. Miatton, G. K. Tran, K. Takinami, A. De Domenico, E. Calvanese, K. K. Strinati, T. Haustein, K. Sakaguchi, S. Barbarossa et al., "5G-MiEdge: Design, Standardization and Deployment of 5G Phase II Technologies," in Conference on Standards for Communications \& Networking. IEEE, 2017, pp. 1-6.

[6] N. H. Motlagh, M. Bagaa, and T. Taleb .UAV-based IoT platform: A crowd surveillance use case.IEEE Communications Magazine, vol. 55, no. 2, pp. 128-134, 2017.

[7] A. Ceselli, M. Premoli, and S. Secci, Mobile Edge Cloud Network Design Optimization .IEEE/ACM Transactions on Networking, vol. 25, no. 3, pp. 1818-1831, 2017.

[8] Y. Mao, C. You, J. Zhang, K. Huang, and K. B. Letaief, "A Survey on Mobile Edge Computing: The Communication Perspective," Communications Surveys \& Tutorials, vol. 19, no. 4, pp. 2322-2358, 2017.

[9] S. Wang, X. Zhang, Y. Zhang, L. Wang, J. Yang, and W. Wang, “A Survey on Mobile Edge Networks: Convergence of Computing, Caching and Communications,"IEEE Access, vol. 5, pp. 6757-6779, 2017.

[10]C. Vallati, A. Virdis, E. Mingozzi, and G. Stea .Mobile-Edge Computing Come Home Connecting Things in Future Smart Homes Using LTE Device-to-Device Communications. Consumer Electronics Magazine, vol. 5, no. 4, pp. 77-83, 2016.

[11]P. Mach and Z. Becvar .Cloud Aware Power Control for Real-time Application Offloading in Mobile Edge Computing .Transactions on Emerging Telecommunications Technologies, vol. 27, no. 5, pp. 648-661, 2016.

[12]P. Mach and Z. Becvar .Mobile Edge Computing: A Survey on Architecture andComputation Offloading. IEEE Communications Surveys \&amp; Tutorials, vol. 19, no. 3, pp. 1628-1656, 2017.

[13] Y. Sun, S. Zhou, and J. Xu, EMM: Energy-Aware Mobility Management for Mobile EdgeComputing in Ultra Dense Networks. IEEE Journal on Selected Areas in Communications, vol. 35, no. 11, pp. 2637-2646, 2017.

[14]B. Cheng, G. Solmaz, F. Cirillo, E. Kovacs, K. Terasawa, and A. Kitazawa.FogFlow: EasyProgramming of IoT Services Over Cloud and Edges for Smart Cities," vol. 5, no. 2, pp. 696-707, 2018.

[15]R. Roman, J. Lopez, and M. Mambo, "Mobile Edge Computing, Fog et al.: A Survey andAnalysis of Security Threats and Challenges," Future Generation Computer Systems, 2016.

[16] V.D.Ambeth Kumar, G.Saranya ,D.Elangovan, V.RahulChiranjeevi, V.D.Ashok Kumar, .IOT Based Smart Museum Using Wearable Device.Lecture Notes in Networks and Systems, Vol.55, pp: 33-42, 2018(Springer)

[17] V.D.Ambeth Kumar, G.Gokul, S.Malathi, K.Vengatesan, D.Elangovan, B.Chitra, .Implementation Of The Pulse Rhythemic Rate For The Efficient Diagonising Of The Heart Beat.Healthcare Technology Letters (IET) 2019 Apr 17;6(2):48-52. 